American Journal of Environmental Sciences 7 (4): 383-396, 2011

ISSN 1553-345X

(C) 2011 Science Publications

\title{
Developmental Design of Anaerobic Wetland System for Mining Waste Water Treatment
}

\author{
${ }^{1}$ Muhammad Aqeel Ashraf, ${ }^{1}$ Mohd. Jamil Maah and ${ }^{2}$ Ismail Yusoff \\ ${ }^{1}$ Department of Chemistry, \\ ${ }^{2}$ Department of Geology \\ University of Malaya, Kuala Lumpur 50603, Malaysia
}

\begin{abstract}
Problem statement: Tin mining is one of the oldest industries in Malaysia that have started since 1820s. These mining activities have resulted in about 13,700 hectors of tin tailings throughout the peninsula. These tailings have created numerous environmental problems such as threat to natural reserves due to landscape changes, damage to natural drainage, pollution and destruction of natural habitats. Approach: This research provided an approach for designing a constructed wetland system for treatment of tin-contaminated wastewater from mining catchment, a system that is known to provide a more economical treatment than the conventional system. Design of wetland was mostly based on the review of scientific literature, theoretical modelling and verification of performance via a pilot system. Results: Initially, physio-chemical characteristics and concentration of heavy metals in the soil and ponds were evaluated. It was found that the soil and water quality of area is highly degraded. This study will help for the design of the wetland for wastewater treatment. The study area consists of five mined out ponds in the catchment, each pond arranged in series with a $48 \mathrm{~h}$ hydraulic retention time. Wetland system comprises of three compartments in series-an 'inflow' pond receiving untreated tailings water overflowing into a wetland compartment, which in turn overflows into an 'outflow' pond receiving the now treated water. Each compartment filled with approximately $50 \mathrm{~cm}$ depth of a mixture of the cattle manure as $(25 \%)$ and municipal waste compost $(75 \%)$ as substrate. Waterproof baffles in each wetland compartment serve to increase the flow path of the water, thereby increasing the potential for sulphate retention. Additionally 30 tonnes of limestone will be deposited at the far end of the wetland, to facilitate final $\mathrm{pH}$ adjustment if it should be required. On site a computer connected to the pumps regulates the flow of tailings water through the systems. Conclusion: The planting density chosen is based on similar research on constructed wetlands. Proposed anaerobic wetland is first of its kind introduce for mining waste water treatment in Malaysia. Good results have achieved on laboratory scale operations for this system.
\end{abstract}

Key words: Water quality, heavy metals, constructed wetland, wastewater treatment, anaerobic system, physic-chemical characteristics, municipal compost, vegetation characteristic

\section{INTRODUCTION}

Wetlands are defined as having a water table above or at the soil surface for a significant proportion of the year, which is a determining factor in their make-up of the ecosystem, an emergent vegetation characteristic of wet biotopes (often containing a large proportion of helophytes), and a soil characteristic of wet biotopes (anoxic, chemically reduced). In treatment wetlands, contaminated water flows through soil, where biological and physical reactions remove contaminants (Cvetkovic and Chow-Fraser, 2011). Traditionally, treatment wetlands have been used to remove organic and inorganic pollutants from wastewater. So most research pertaining to pollutant removal has been concerned with the biodegredation of organic and inorganic compounds.

A wetland is a more or less engineered system, designed to enhance the interaction between vegetation, fauna, soils and microorganisms for the primary purpose of pollutant removal from agricultural wastewaters (e.g., parlour washings), runoff (e.g., field, road, farmyard) or sewage (Knight et al., 2000; Ghaly et al., 2011; Michailidis, 2006). In wetland systems, water flows vertically or/and horizontally through a porous substrate (e.g., gravel, sand) planted with

Corresponding Author: Muhammad Aqeel Ashraf, Department of Chemistry, University of Malaya, Kuala Lumpur 50603, Malaysia 
macrophytes. Normally wetlands are composed of one or more shallow, several cells of variable depth and characteristics, (depth 30-40 $\mathrm{cm}$ ) through the vegetation, made of submerged, emergent or floatingleaved plants, which is designed to receive and treat contaminated surface water runoff from mining lakes and ponds, in such a manner that any discharge from the wetland will not pollute the water environment (Kadlec et al., 2010; Steer et al., 2003).

Wetlands are attractive as an endpoint in the rehabilitation of mine wastes, such as tailings and tailings water, for two reasons. First, pollutants originating from mining activities, such as metals and sulphur, are relatively immobile when present under waterlogged conditions (Zhang et al., 2010). Second, pollutants are retained by the wetlands from water passing through the wetlands (Jitrapinate et al., 2011; Keita et al., 2009). Both characteristics are largely due to the same processes. Permanently waterlogged wetland soils are generally anaerobic, because of the relatively low diffusion rate of oxygen through water compared to air. In addition, micro-organisms present in such soils respire using terminal electron acceptors other than oxygen. Such organisms can, for example, reduce ferric iron to its ferrous form, or reduce sulphate to sulphide. The formation of highly insoluble sulphide from soluble sulphate in particular is important. Not only does that process lead to the precipitation of sulphur, but also co-precipitation of metals, including iron, zinc, lead and cadmium. Once metal sulphides have precipitated, they are stable and insoluble providing the soil remains anaerobic (Lang et al., 2008). Wetlands can therefore be used in several aspects of rehabilitation of mine wastes. First, mine tailings can be revegetated under wetland conditions, using wetland plants, and second, the quality of water originating from mining operations can be improved by passing it through wetlands, whether they are naturallyoccurring or constructed specifically for that purpose (Asadi et al., 2009).

Constructed wetlands have several advantages if properly designed (Kangrang and Chaleeraktrakoon, 2007; Reza, 2008; Al-Hamed et al., 2010): they can provide high and consistent level of treatment for nutrients, pathogens and hydrocarbons, contribute to runoff and flood management if built large enough, act as long-term carbon stores, are easy to manage, require little maintenance and energy use and are cheaper than alternative methods for farm runoff disposal. They minimize odours produced by agricultural wastes, due to their dense plant cover and shallow surface flow, are aesthetically pleasing if designed in a sensible manner, bring additional value to farmland and enhance habitat and biodiversity. They can be used as contingency measures against accidental spillages, for irrigation if large enough and they reduce the need for dirty water storage, decrease land area needed for application and allow better timing of land spreading.

Constructed wetlands have some limitations: their construction requires relatively large areas in comparison with conventional treatment systems and they can be costly and in the long-term and may be reduced when pollutants enter rapidly and in large amounts, and they require a minimum of water to maintain ecosystem function (Kadlec, 2006). Moreover, the creation and mismanagement of wetlands may alter existing wetlands or local hydrology, e.g. creating a pathway between the farm and water body where it was previously inexistent, can introduce invasive species, disrupt and intoxicate plant and animal communities (Heryati et al., 2011; Galbrand et al., 2008).

Wetlands performance varies strongly spatially and temporally, and wetlands may act as sinks or sources of contaminants, depending on their age, location, design, wastewater characteristics, loadings, retention time, hydrological conditions, season, biological activity and management (Ahmadpour et al., 2010).

Vegetation in wetlands (Phragmites australis, Typha latifolia or Scirpus spp.) has an overall positive impact on treatment efficiency: it stabilizes the surface of the wetland, reduces flow velocity and facilitates sedimentation, takes up nutrients from sediment and stores them in green parts or other organs (roots, tubers), adsorbs metals, provides fixation sites for microorganisms, conducts oxygen to sediment, produces aerobic conditions which enhance nitrification, and provides wildlife with habitat and food (Nasipuri et al., 2010; Knowles et al., 2011). Plant nutrient uptake is not the major pathway for $\mathrm{N}$ and $\mathrm{P}$ removal but can contribute $16-75 \%$ removal of total nitrogen and $12-73 \%$ removal of total phosphorus. An appropriate plant selection can improve wetland efficiency: plants should be native, perennial, highly productive for rapid nutrient uptake, produce rhizome or storage organs, and be tolerant to high pollutant loads and anaerobic conditions (Vymazal, 2009). However, dying plants and accumulation of debris might increase BOD, decrease dissolved oxygen or release nutrients and affect treatment performance. Vegetation removal can be a way to export nutrients from the wetland, but it is costly, time-consuming and may disturb wetland function and decrease efficiency (Whigham, 1999). 
Heavy metals (e.g., from oil spillages, mining) may be removed or stored by sedimentation, adsorption to plants and sediment, plant uptake, biological assimilation, decomposition, chemical transformation and volatilisation, these processes being mainly influenced by temperature, $\mathrm{pH}$, redox potential and availability of adsorption sites (Faulwetter et al., 2008; Turner et al., 2000).

The variability in the design, use and performance of wetlands, and the lack of detailed studies investigating simultaneously the hydrology, ecology and economics of individual systems justifies the necessity to explore the efficiency, limitations and sustainability of the particular design used in Malaysia until now.

Wetland plants for vegetation of mine tailings: In Malaysia the approach for revegetation of mine tailings has not yet been applied, but has been proposed for the Bestari Jaya catchment. Malaysia has a net precipitation level greater than the evapotranspiration level, therefore the supply of water for the establishment of wetlands should not pose a problem. However, characteristically, mine tailings have a low nutrient content and high concentrations of potentially toxic metals and sulphur compounds, both of which can be problematic for the successful establishment of plants. Nutrient supply to the plants can be improved by adding fertiliser. Alternatively, plants that have low nutrient re- quirements can be used. The latter solution is more attractive as it reduces the cost of the reclamation process. In addition, plants that are used for revegetation purposes can survive higher metal concentrations than plants that are not accustomed to such conditions. Brooks observed that the amphibious floating sweetgrass (Glyceria fluitans) was growing very well on tailings in a pond near the abandoned lead-zinc mine at Glendalough, Co. Wicklow (Brooks et al., 2005). This was the first time that this species was reported to grow under such conditions and a study was initiated to investigate whether the species was suitable for revegetation purposes (Spieles and Mitsch, 1999).

Filtering of metals from contaminated water passing through a 'volunteer' wetland: Wetlands can also be used for quality improvement of contaminated water (Tilley and Brown, 1998).

Biogeochemical and physical processes, as well as uptake by plants, lead to reduced concentrations of contaminants, including nitrogen, phosphorus and metals, as the water passes through the wetlands. Naturally occurring, so-called 'volunteer' wetlands, as well as constructed wetlands, can be used for the treatment of polluted water. Many studies have shown the effectiveness of such systems in reducing concentrations of contaminants in water, but the question still remains as to how the system itself and its longevity are affected by the accumulation of toxic substances (Amezaga et al., 2002). If treatment wetlands deteriorate within a relatively short period of time (10-20 years) then this approach would not be attractive for municipal and industrial purposes. Most constructed treatment wetlands are younger than fifteen years and, therefore, have not been active long enough for an accurate assessment of the impact of accumulation of pollutants on their longevity. Natural, volunteer wetlands may have been receiving pollutants for a much longer period of time. Such situations are rather rare, but one example exists at Glendalough, Co. Wicklow.

A key objective of this feasibility study was to design a treatment system that would be inexpensive in terms of both initial installation costs and long-term operating and maintenance costs.

Study area: Bestari Jaya catchment is located at $3^{\circ}, 24^{\prime}$ $40.41^{\circ}$ " $\mathrm{N}$ and $101^{\circ} 24^{\prime} 56.23^{\prime}$ ' E. It is a part of Kuala Selangor district, located in Selangor, biggest state of the country. District Kuala Selangor has three main towns namely, Mukim Batang Berjuntai, Mukim Ulu Tinggi, Mukim Tg.karang. Bestari Jaya is located in Mukim Batang Berjuntai (Ashraf et al., 2011a). Tin mining activities has ceased from last ten years, now sand mining. The catchment has total of 442 small and big mining lakes and ponds (Fig. 1). Bestari Jaya has a tropical, humid climate, with very little variations in temperature throughout the year. The average temperature of the area is $32^{\circ} \mathrm{C}$ during day and $23^{\circ} \mathrm{C}$ at night (Ashraf et al., 2011b).The Bestari Jaya catchment is strongly impacted by mining pollution, which affects Selangor River as mining water flows freely without treatment to the river via small connecting River Ayer Hitam.

The protection of the River Selangor is a high priority due to its high ecological value and economic importance, in particular for drinking purposes and fishing which represents a significant local source of income and employment. In order to address mining pollution in the Bestari Jaya Catchment, the construction of wetlands was suggested and promoted by the writer. 


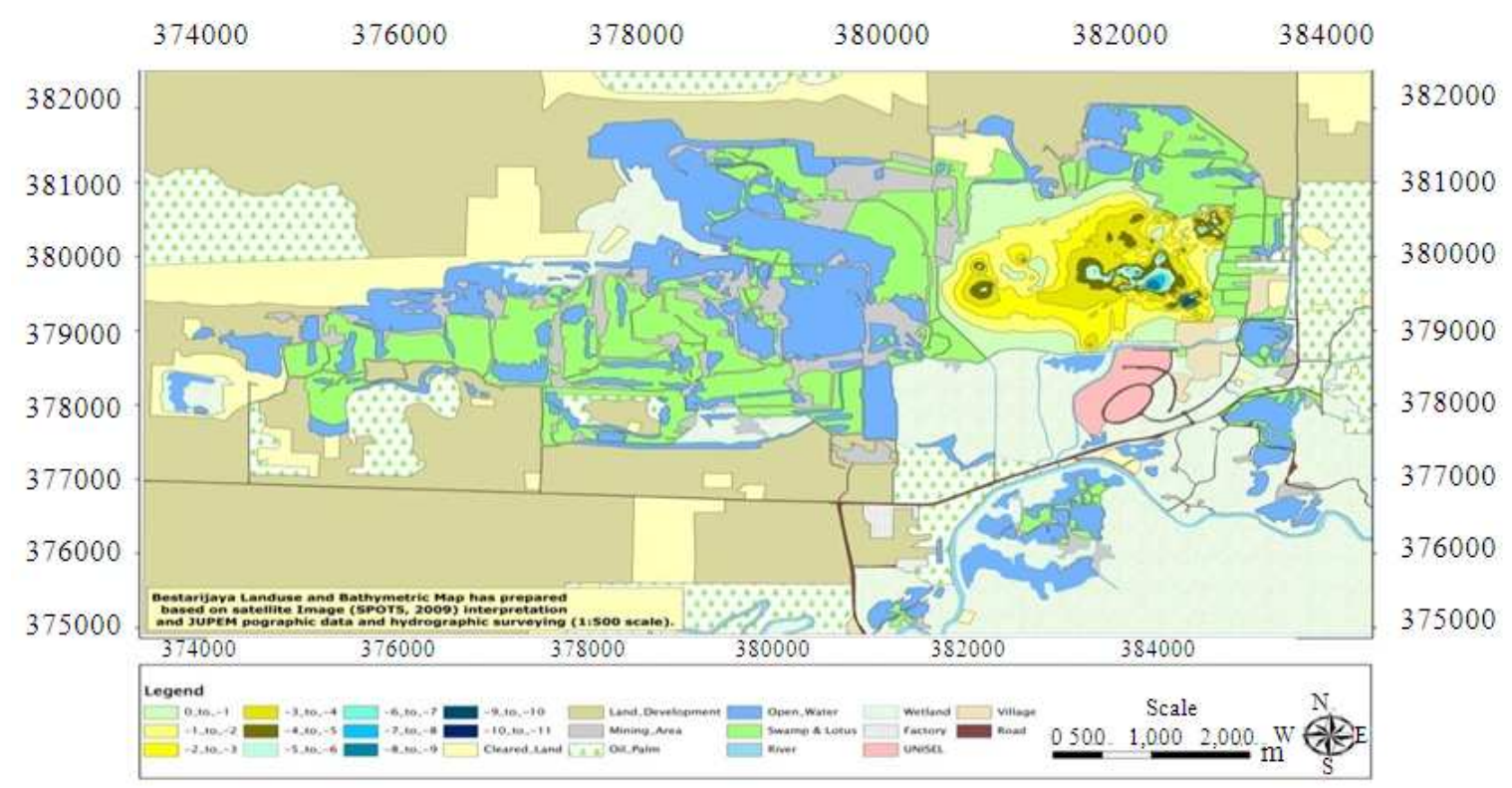

Fig. 1: Bestari Jaya Catchment showing selected ponds (Yellow) for wetland development

\section{MATERIALS AND METHODS}

Sampling: Total 92 hectors of downstream part of the catchment were sampled, starting from north-eastern side of catchment to Sungai Ayer Hitam that meets Sungai Selangor at the Jalan Timur Tambahan road junction. Water samples were taken from two exmining ponds, at the junction of Sungai Ayer Hitam and at the junction of Sungai Selangor and soil samples were taken at the embankment of the river and ponds and the area nearby. Global Positioning System GPS was used to determine the actual coordinates of the sampling sites and to reconfirm the location of the sampling site during subsequent sampling periods. Soil and water investigation consists of ten locations, in order to determine and to provide ground information for subsequent detailed planning of the future work. For soil sampling multiple sub samples were taken from each location and then samples were homogenized into composite sample with stainless spoon and then sub sampled by spoon into each sample container to get accurate results. For ex-situ analysis, soil samples were collected from first $20 \mathrm{~cm}$ of the soil in polythene bags and water samples were collected $10 \mathrm{~cm}$ below the surface water using HDPE bottle $500 \mathrm{~mL}$ (Noble et al., 2011). The water samples were preserved by few drops of nitric acid (70\%) and stored in an icebox and transported to laboratory for analysis.
Water Investigation: Two ponds P1 and P2 at downstream of the catchment were investigated for physio-chemical parameters and heavy metals analysis. Physio-chemical parameters were analysed by instrument Hydro lab HACH MS5 while colour of water is measured by True Colour Units (TCU). For quantitave estimation of heavy metals, samples were digested by acid digestion method (ASTM D 5198-09) and analyzed by atomic absorption spectrophotometer.

Soil Investigation: Soil physico-chemical parameters measured were soil texture, temperature, hydraulic conductivity, moisture content, soil $\mathrm{pH}$, and soil grain size. Texture is determined by (Simonit et al., 2005), soil temperature by soil thermometer, hydraulic conductivity by (ASTM D5084-03) method, moisture content by gravimetric method, soil $\mathrm{pH}$ was measured by potentiometrically and Soil grain size was measured by (ASTM D422) method. For estimation of heavy metals the samples were air dried, crushed in a mortar pestle and sieved up to $0.5 \mathrm{~mm}$ mesh sieve and then digested by wet digestion method and analysed by a Perkin Elmer AAnalyst 800 atomic absorption spectrophotometer.

\section{RESULTS}

Water quality parameters for 15 sampling stations are shown in (Table 2). Results shows that there is variation in water quality at all sampling stations. 
Am. J. Environ. Sci., 7 (4): 383-396, 2011

Table 1: Construction materials quantities and estimated costs for the Bestari Jaya wetland

\begin{tabular}{llc}
\hline Quantity/ & $\begin{array}{l}\text { Estimated } \\
\text { duration }\end{array}$ & Cost RM \\
\hline Material & $614 \mathrm{t}$ & 10000 \\
\hline Pulverised Fuel Ash PFA & $60 \mathrm{t}$ & 2000 \\
Cattle Manure $^{\mathrm{a}}$ & $49 \mathrm{t}$ & 2000 \\
Municipal Waste Compost & $31 \mathrm{t}$ & 3000 \\
Lime stone & $11 \mathrm{~T}$ & 1000 \\
Pea gravel & $10 \mathrm{t}$ & 1000 \\
Broken stone & - & 5000 \\
Pipe working/building material & $64 \mathrm{t}$ & 5000 \\
Top Soil & 6 months & 8000 \\
Design engineering/consultation & 7 weeks & 20000 \\
Plant hire and operation & & $57000 \mathrm{RM}$ \\
Total & &
\end{tabular}

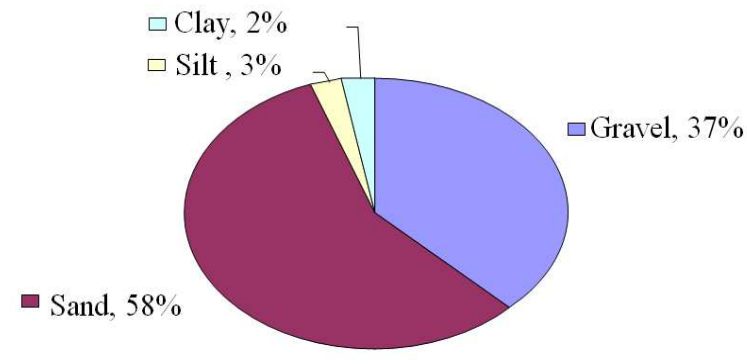

$\square$ Gravel $\square$ Sand $\square$ Silt $\square$ Clay

Fig. 2: Soil profile in the study area

Water quality parameters of sampling station WS15 are; colour $9 \mathrm{TCU}$, temperature $32.51^{\circ} \mathrm{C}, \mathrm{pH} 5$, conductivity $1756 \mu \mathrm{mhos} / \mathrm{cm}$, salinity $0.30 \%$, turbidity

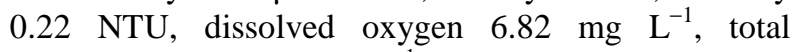
dissolved solids $2998 \mathrm{mg} \mathrm{L}^{-1}$ while at WS1 (Junction of Sungai Ayer Hitam + Sungai Selangor) water quality parameters are; colour $5 \mathrm{TCU}$, temperature $32.19^{\circ} \mathrm{C}$, pH 6.47, conductivity $1640 \mu \mathrm{mhos} / \mathrm{cm}$, salinity $0.26 \%$, turbidity $0.12 \mathrm{mg} \mathrm{L}^{-1}$ dissolved Oxygen $6.59 \mathrm{mg} \mathrm{L}^{-1}$, total dissolved solids $2654 \mathrm{mg} \mathrm{L}^{-1}$. This shows variation trends at all sampling stations are from upstream to downstream.

Table 3 and 4, (Fig. 2) show the physico-chemical properties of soil. (Table 3) shows the that average contents of the soil are gravel $37.3 \%$ with diameter 3-6 $\mathrm{mm}$, sand $57.20 \%$ with diameter $0.1-2 \mathrm{~mm}$, silt $2.9 \%$ with diameter $0.008-0.4 \mathrm{~mm}$ and clay $2.46 \%$ with diameter $0.0008-0.0014 \mathrm{~mm}$. (Table 4) indicates that the average moisture content of soil is $6.36 \%$ of soil, temperature $22.0^{\circ} \mathrm{C}^{\prime} \mathrm{pH} 5.64$ and hydraulic conductivity is $13.7 \mathrm{~cm} \mathrm{day}^{-1}$.
Contribution of heavy metals in water

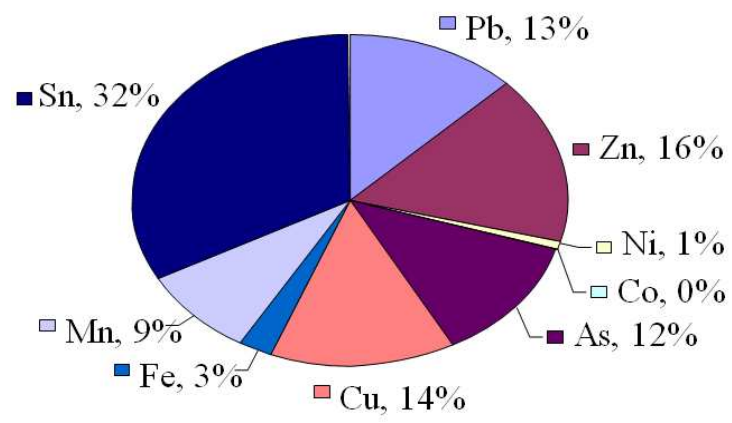

$\square \mathrm{Pb} \square \mathrm{Zn} \square \mathrm{Ni} \square \mathrm{Co} \backsim \mathrm{As} \square \mathrm{Cu} \square \mathrm{Fe} \square \mathrm{Mn} \backsim \mathrm{Sn}$

Fig. 3: Contribution of heavy metals in water

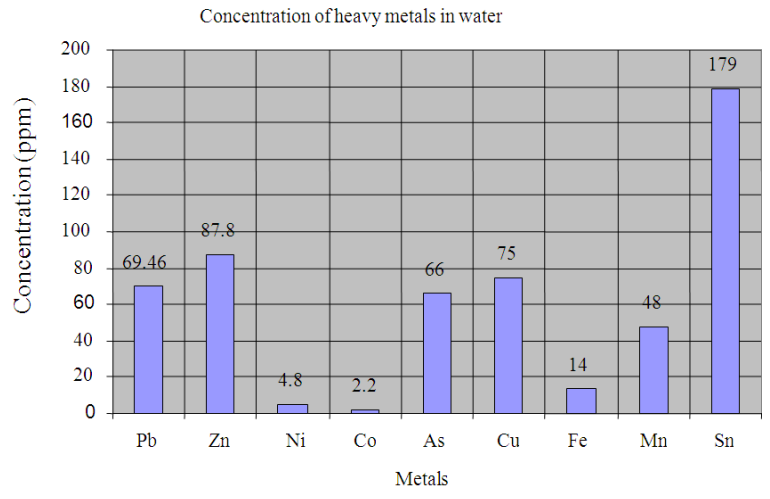

Fig. 4: Concentration of heavy metals in water

Metal concentration of water and soil are good indicators of degree of contamination. (Tabl 4) indicates physo-chemical characcteristics of soil at Bestari Jaya catchment. (Table 5), (Fig. 3 and 4) show the concentration of heavy metals in water of the area under investigation. Similarly (Table 6), (Fig. 5 and 6) show the concentration of heavy metals in soil which is realy alrming. At the sampling station WS1 are as follows; lead 38, zinc 88, nickel 2.5, cobalt 1.0, arsenic 30, copper 59, iron 06, manganese 44 and tin $85 \mathrm{mg} \mathrm{L}^{-1}$ while at sampling station WS15 concentration of heavy metals are as follows; lead 96, zinc 121 nickel 2.8, cobalt 1.8 , arsenic 77 , copper 80 , iron 16 , manganese 48 and tin $250 \mathrm{mgL}^{-1}$. Same variation trends of decrease in metal concentration are at all sampling stations from upstream to downstream. 
Am. J. Environ. Sci., 7 (4): 383-396, 2011

Table 2: Physio-chemical parameters of surface water in the study area

\begin{tabular}{|c|c|c|c|c|c|c|c|c|c|c|}
\hline Sample No. & Location & Coordinates & $\begin{array}{l}\text { Colour } \\
\text { TCU }\end{array}$ & $\begin{array}{l}\text { Temperature } \\
\mathrm{C}^{\circ}\end{array}$ & $\mathrm{pH}$ & $\begin{array}{l}\text { Conductivity } \\
\mu \mathrm{S} / \mathrm{cm}\end{array}$ & $\begin{array}{l}\text { Salinity } \\
\%\end{array}$ & $\begin{array}{l}\text { Turbidity } \\
\text { NTU }\end{array}$ & $\begin{array}{ll}\text { Dissolved } & \text { Total D } \\
\text { Oxygen mg/L } & \text { Solids } \\
\end{array}$ & $\begin{array}{l}\text { issolved } \\
\mathrm{mg} / \mathrm{L}\end{array}$ \\
\hline WS1 & $\begin{array}{l}\text { Junction of Sungai } \\
\text { Selangor }+ \text { Sungai Ayer Hitam }\end{array}$ & $\begin{array}{l}3^{0} 24^{\prime} 28.04^{\prime \prime} \mathrm{N} \\
101^{0} 25^{\prime} 54.89^{\prime \prime} \mathrm{E}\end{array}$ & 5.00 & 32.19 & 6.47 & 1640.00 & 0.2700 & 16.00 & 6.83 & 2654.00 \\
\hline WS2 & $\begin{array}{l}\text { Junction of Sungai } \\
\text { Ayer Hitam + Sungai Udang }\end{array}$ & $\begin{array}{l}3^{0} 24^{\prime} 30.96^{\prime \prime} \mathrm{N} \\
101^{0} 25^{\prime} 54.08^{\prime \prime} \mathrm{E}\end{array}$ & 7.00 & 32.62 & 6.27 & 1680.00 & 0.2800 & 18.00 & 6.65 & 2784.00 \\
\hline WS3 & $\begin{array}{l}\text { Sungai Ayer } \\
\text { Hitam water flow }\end{array}$ & $\begin{array}{l}3^{0} 24^{\prime} 36.54^{\prime \prime} \mathrm{N} \\
101^{0} 25^{\prime} 59.96^{\prime \prime} \mathrm{E}\end{array}$ & 7.00 & 32.52 & 6.27 & 1686.00 & 0.2800 & 18.00 & 6.70 & 2797.00 \\
\hline WS4 & $\begin{array}{l}\text { Sungai Ayer } \\
\text { Hitam at bank of UNISEL }\end{array}$ & $\begin{array}{l}3^{0} 24^{\prime} 56.68^{\prime \prime} \mathrm{N} \\
101^{0} 25^{\prime} 59.18^{\prime \prime} \mathrm{E}\end{array}$ & 7.00 & 32.51 & 6.29 & 1686.00 & 0.2800 & 18.00 & 6.72 & 2800.00 \\
\hline WS5 & $\begin{array}{l}\text { Sungai Ayer } \\
\text { Hitam at bank of UNISEL }\end{array}$ & $\begin{array}{l}3^{0} 25^{\prime} 06.69^{\prime \prime} \mathrm{N} \\
101^{0} 26^{\prime} 08.14^{\prime \prime} \mathrm{E}\end{array}$ & 7.00 & 32.98 & 6.29 & 1688.00 & 0.2800 & 19.00 & 6.15 & 2812.00 \\
\hline WS6 & $\begin{array}{l}\text { Junction of Pond } 1 \\
\text { to Sungai Ayer Hitam }\end{array}$ & $\begin{array}{l}3^{0} 25^{\prime} 16.57^{\prime \prime} \mathrm{N} \\
101^{0} 26^{\prime} 09.24^{\prime \prime} \mathrm{E}\end{array}$ & 7.00 & 32.90 & 6.28 & 1690.00 & 0.2800 & 18.00 & 6.42 & 2864.00 \\
\hline WS7 & Pond 1 & $\begin{array}{l}3^{0} 25^{\prime} 13.37^{\prime \prime} \mathrm{N} \\
101^{0} 26^{\prime} 04.66^{\prime \prime} \mathrm{E}\end{array}$ & 7.00 & 32.75 & 5.78 & 1704.00 & 0.2900 & 19.00 & 6.34 & 2900.00 \\
\hline WS8 & Pond 1 & $\begin{array}{l}3^{0} 25^{\prime} 15.56^{\prime \prime} \mathrm{N} \\
101^{0} 26^{\prime} 0.79^{\prime \prime} \mathrm{E}\end{array}$ & 7.00 & 32.50 & 5.2 & 1744.00 & 0.2900 & 20.00 & 6.00 & 2934.00 \\
\hline WS9 & $\begin{array}{l}\text { Junction of Pond } 1 \\
\text { to another Pond }\end{array}$ & $\begin{array}{l}3^{0} 25^{\prime} 13.87^{\prime \prime} \mathrm{N} \\
101^{0} 25^{\prime} 55.35^{\prime \prime} \mathrm{E}\end{array}$ & 7.00 & 32.44 & 5.32 & 1734.00 & 0.2900 & 19.00 & 6.42 & 2924.00 \\
\hline WS 10 & $\begin{array}{l}\text { Junction of Sungai } \\
\text { Ayer Hitam to Pond } 2 \\
\text { at north-eastern boundary UNISEL }\end{array}$ & $\begin{array}{l}3^{0} 25^{\prime} 20.92^{\prime \prime} \mathrm{N} \\
101^{0} 26^{\prime} 12.06^{\prime \prime} \mathrm{E}\end{array}$ & 7.00 & $\begin{array}{l}32.28 \\
32.32\end{array}$ & $\begin{array}{l}5.41 \\
5.34\end{array}$ & $\begin{array}{l}1694.00 \\
1710.00\end{array}$ & $\begin{array}{l}0.2800 \\
0.2800\end{array}$ & $\begin{array}{l}18.00 \\
20.00\end{array}$ & $\begin{array}{l}6.39 \\
6.28\end{array}$ & $\begin{array}{l}2887.00 \\
2912.00\end{array}$ \\
\hline WS11 & $\begin{array}{l}\text { Junction of Sungai } \\
\text { Ayer Hitam to Pond } 2\end{array}$ & $\begin{array}{l}3^{0} 25^{\prime} 22.11^{\prime \prime} \mathrm{N} \\
101^{0} 26^{\prime} 6.66^{\prime \prime} \mathrm{E}\end{array}$ & 9.00 & 32.12 & 5.22 & 1724.00 & 0.2900 & 22.00 & 6.87 & 2920.00 \\
\hline WS12 & Pond 2 & $\begin{array}{l}3^{0} 25^{\prime} 22.54^{\prime \prime} \mathrm{N} \\
101^{0} 26^{\prime} 0.94^{\prime \prime} \mathrm{E}\end{array}$ & 9.00 & & & & & & & \\
\hline WS13 & Pond 2 & $\begin{array}{l}3^{0} 25^{\prime} 22.05^{\prime \prime} \mathrm{N} \\
101^{0} 25^{\prime} 58.38^{\prime \prime} \mathrm{E}\end{array}$ & 9.00 & 32.57 & 5.39 & 1732.00 & 0.2900 & 24.00 & 6.45 & 2922.00 \\
\hline WS 14 & Pond2 & $\begin{array}{l}3^{0} 25^{\prime} 23.71^{\prime \prime} \mathrm{N} \\
101^{0} 25^{\prime} 52.42^{\prime \prime} \mathrm{E}\end{array}$ & 9.00 & 32.29 & 5.28 & 1738.00 & 0.2900 & 22.00 & 6.59 & 2956.00 \\
\hline WS15 & $\begin{array}{l}\text { Junction of Pond } 2 \\
\text { to another Pond } \\
\text { Standard Deviation }\end{array}$ & $\begin{array}{l}3^{0} 25^{\prime} 33.21^{\prime \prime} \mathrm{N} \\
101^{0} 25^{\prime} 51.34 \text { " E }\end{array}$ & 9.00 & 32.51 & 5.00 & 1756.00 & 0.3000 & 22.00 & 6.82 & 2998.00 \\
\hline Mean & Variance (Standard Deviation) & $\begin{array}{l}\mathrm{X} \\
\mathrm{O}^{\prime} \\
\mathrm{O}^{2}\end{array}$ & $\begin{array}{l}7.00 \\
1.18 \\
1.40\end{array}$ & $\begin{array}{r}32.50 \\
0.24 \\
0.06\end{array}$ & $\begin{array}{l}5.71 \\
0.52 \\
0.27\end{array}$ & $\begin{array}{r}1707.00 \\
30.83 \\
950.78\end{array}$ & $\begin{array}{l}0.28000 \\
0.00700 \\
0.00006\end{array}$ & $\begin{array}{l}0.190 \\
2.130 \\
4.552\end{array}$ & $\begin{array}{l}6.50 \\
0.25 \\
0.06\end{array}$ & $\begin{array}{r}2870.00 \\
87.26 \\
7615.49\end{array}$ \\
\hline
\end{tabular}

Table 3: Grain size analysis of the soil

\begin{tabular}{|c|c|c|c|c|c|c|c|c|c|c|c|c|}
\hline $\begin{array}{ll}\text { Sample } & \text { Diameter } \\
\text { No. } & \text { Location }\end{array}$ & $\begin{array}{l}\text { Clay } \\
\text { Coordinates }\end{array}$ & $\begin{array}{l}\text { Gravel } \\
\text { Particle } \\
\text { Diameter } \\
\mathrm{mm}\end{array}$ & $\%$ age & $\begin{array}{l}\text { Course to } \\
\text { Particle } \\
\text { Diameter } \\
\mathrm{mm}\end{array}$ & $\begin{array}{l}\text { Medium } \\
\% \text { age }\end{array}$ & $\begin{array}{l}\text { Total } \\
\mathrm{mm}\end{array}$ & $\begin{array}{l}\text { Sand } \\
\text { Particle } \\
\text { Diameter } \\
\% \text { age }\end{array}$ & $\begin{array}{l}\text { Fine } \\
\text { Particle } \\
\text { Diameter } \\
\% \text { age }\end{array}$ & $\mathrm{mm}$ & $\%$ age & $\begin{array}{l}\text { Silt } \\
\text { Particle } \\
\mathrm{mm}\end{array}$ & $\%$ age \\
\hline $\begin{array}{l}\text { SS1Junction of Jalan Timur } \\
\text { Tambahan + Sungai Selangor }\end{array}$ & $\begin{array}{l}3^{0} 24^{\prime} 29.80^{\prime \prime} \mathrm{N} \\
101^{0} 25^{\prime} 55.08^{\prime \prime} \mathrm{E}\end{array}$ & 4.00 & 37.66 & 1.00 & 32.01 & 0.100 & 25.34 & 57.35 & 0.040 & 2.81 & 0.0014 & 2.18 \\
\hline $\begin{array}{l}\text { SS2Bank of Sungai Ayer } \\
\text { Hitam + Sungai Udang }\end{array}$ & $\begin{array}{l}3^{0} 24^{\prime} 32.03 " \mathrm{~N} \\
101^{0} 25^{\prime} 54.75^{\prime \prime} \mathrm{E}\end{array}$ & 5.00 & 37.98 & 2.00 & 30.45 & 0.200 & 26.73 & 57.18 & 0.020 & 2.40 & 0.0009 & 2.44 \\
\hline SS3Bank of Sungai Ayer Hitam & $\begin{array}{l}3^{0} 24^{\prime} 36.29^{\prime \prime} \mathrm{N} \\
101^{0} 25^{\prime} 57.34^{\prime \prime} \mathrm{E}\end{array}$ & 3.00 & 35.44 & 1.00 & 29.48 & 0.100 & 27.52 & 57.00 & 0.020 & 3.88 & 0.0008 & 3.68 \\
\hline SS4South-eastern boundary of UNISEL & $\begin{array}{l}3^{0} 24^{\prime} 54.73^{\prime \prime} \mathrm{N} \\
101^{0} 26^{\prime} 0.48^{\prime \prime} \mathrm{E}\end{array}$ & 6.00 & 38.41 & 2.00 & 30.18 & 0.300 & 27.36 & 57.54 & 0.040 & 2.57 & 0.0016 & 1.48 \\
\hline $\begin{array}{l}\text { SS5Wetlands developed by } \\
\text { overflow of Pond } 1\end{array}$ & $\begin{array}{l}3^{0} 25^{\prime} 09.78^{\prime \prime} \mathrm{N} \\
101^{0} 25^{\prime} 59.41^{\prime \prime} \mathrm{E}\end{array}$ & 5.00 & 36.98 & 1.00 & 31.24 & 0.300 & 25.57 & 56.81 & 0.010 & 2.88 & 0.0016 & 3.38 \\
\hline SS6Bank of Pond 1 & $\begin{array}{l}3^{0} 25^{\prime} 11.54^{\prime \prime} \mathrm{N} \\
101^{0} 26^{\prime} 07.44^{\prime \prime} \mathrm{E}\end{array}$ & 4.00 & 37.52 & 2.00 & 31.12 & 0.200 & 26.6 & 57.72 & 0.009 & 2.67 & 0.0019 & 2.09 \\
\hline SS7North-eastern boundary of UNISEL & $\begin{array}{l}3^{0} 25^{\prime} 13.40^{\prime \prime} \mathrm{N} \\
101^{0} 26^{\prime} 11.64^{\prime \prime} \mathrm{E}\end{array}$ & 3.00 & 37.84 & 2.00 & 30.52 & 0.200 & 25.92 & 56.44 & 0.020 & 3.13 & 0.0018 & 2.59 \\
\hline $\begin{array}{l}\text { SS8Wetlands developed by } \\
\text { overflow of Pond } 1\end{array}$ & $\begin{array}{l}3^{0} 25^{\prime} 59.18^{\prime \prime} \mathrm{N} \\
101^{0} 25^{\prime} 56.90^{\prime \prime} \mathrm{E}\end{array}$ & 4.00 & 36.85 & 2.00 & 29.92 & 0.200 & 26.34 & 56.26 & 0.008 & 3.98 & 0.0019 & 2.91 \\
\hline $\begin{array}{l}\text { SS9Junction of Sungai Ayer Hitam } \\
\text { with pond } 1 \text { on north-western side }\end{array}$ & $\begin{array}{l}3^{0} 25^{\prime} 19.80^{\prime \prime} \mathrm{N} \\
101^{0} 26^{\prime} 13.07^{\prime \prime} \mathrm{E}\end{array}$ & 5.00 & 35.94 & 2.00 & 30.74 & 0.100 & 26.4 & 57.14 & 0.100 & 3.76 & 0.0016 & 3.16 \\
\hline $\begin{array}{l}\text { SS10Junction of Sungai Ayer Hitam } \\
\text { with pond } 1 \text { on south-western side }\end{array}$ & $\begin{array}{l}3^{0} 25^{\prime} 22.79^{\prime \prime} \mathrm{N} \\
101^{0} 26^{\prime} 11.06^{\prime \prime} \mathrm{E}\end{array}$ & 5.00 & 37.72 & 2.00 & 29.12 & 0.100 & 27.86 & 56.98 & 0.300 & 2.81 & 0.0008 & 2.49 \\
\hline SS 11 Wetland between Pond 1 and Pond 2 & $\begin{array}{l}3^{0} 25^{\prime} 20.64 ” \mathrm{~N} \\
101^{0} 25^{\prime} 54.37^{\prime \prime} \mathrm{E}\end{array}$ & 4.00 & 37.44 & 1.00 & 31.19 & 0.300 & 27.15 & 58.34 & 0.400 & 2.21 & 0.0008 & 2.01 \\
\hline SS12Embankment of Pond 2 & $\begin{array}{l}3^{0} 25^{\prime} 27.52 ” \mathrm{~N} \\
101^{0} 25,53.89^{\prime \prime} \mathrm{E}\end{array}$ & 3.00 & 37.74 & 1.00 & 32.92 & 0.200 & 25.47 & 58.39 & 0.200 & 2.10 & 0.0009 & 1.77 \\
\hline SS13Embankment of Pond 2 & $\begin{array}{l}3^{0} 25^{\prime} 22.86 " \mathrm{~N} \\
101^{0} 25^{\prime} 51.67^{\prime \prime} \mathrm{E}\end{array}$ & 6.00 & 37.54 & 2.00 & 31.44 & 0.400 & 26.13 & 57.57 & 0.200 & 2.56 & 0.0014 & 2.33 \\
\hline SS14Embankment of Pond 2 & $\begin{array}{l}3^{0} 25^{\prime} 34.95^{\prime \prime} \mathrm{N} \\
101^{0} 25^{\prime} 49.93^{\prime \prime} \mathrm{E}\end{array}$ & 3.00 & 37.75 & 2.00 & 30.88 & 0.300 & 25.71 & 56.59 & 0.300 & 3.12 & 0.0016 & 2.54 \\
\hline SS15Embankment of Pond 2 & $\begin{array}{l}3^{0} 25^{\prime} 36.24 " \mathrm{~N} \\
101^{0} 25^{\prime} 52.14^{\prime \prime} \mathrm{E}\end{array}$ & 5.00 & 37.58 & 1.00 & 31.12 & 0.200 & 26.76 & 57.88 & 0.009 & 2.62 & 0.0018 & 1.92 \\
\hline Mean & $\mathrm{X}$ & 4.33 & 37.35 & 1.60 & 30.82 & 0.200 & 26.45 & 57.20 & 0.110 & 2.90 & 0.0013 & 2.46 \\
\hline Standard Deviation & O & 1.04 & 0.77 & 0.50 & 0.95 & 0.090 & 0.78 & 0.63 & 0.130 & 0.57 & 0.0004 & 0.61 \\
\hline Variance (Standard Deviation) & $\mathrm{O}^{2}$ & 1.09 & 0.60 & 0.25 & 0.91 & 0.008 & 0.61 & 0.40 & 0.010 & 0.33 & 0.0000 & 0.37 \\
\hline
\end{tabular}


Am. J. Environ. Sci., 7 (4): 383-396, 2011

Table 4: Physio-chemical parameters of soil in the study area

\begin{tabular}{|c|c|c|c|c|c|c|}
\hline \multicolumn{2}{|l|}{ Sample } & Coordinates & \multicolumn{2}{|c|}{ Moisture Content (\%) } & \multicolumn{2}{|c|}{ Hydraulic conductivity } \\
\hline SS1 & $\begin{array}{l}\text { Junction of Jalan Timur } \\
\text { Tambahan + Sungai Selangor }\end{array}$ & $\begin{array}{l}3^{0} 24^{\prime} 29.80^{\prime \prime} \mathrm{N} \\
101^{0} 25,55.08^{\prime \prime} \mathrm{E}\end{array}$ & 6.230 & 21.22 & 5.70 & 14.30 \\
\hline SS2 & $\begin{array}{l}\text { Bank of Sungai Ayer Hitam } \\
+ \text { Sungai Udang }\end{array}$ & $\begin{array}{l}3^{0} 24^{\prime} 32.03 " \mathrm{~N} \\
101^{0} 25,54.75 " \mathrm{E}\end{array}$ & 6.240 & 22.84 & 5.50 & 12.40 \\
\hline SS3 & $\begin{array}{l}\text { Bank of Sungai } \\
\text { Ayer Hitam }\end{array}$ & $\begin{array}{l}3^{0} 24^{\prime} 36.29 " \mathrm{~N} \\
101^{0} 25,57.34^{\prime \prime} \mathrm{E}\end{array}$ & 6.380 & 21.44 & 5.30 & 15.20 \\
\hline SS4 & $\begin{array}{l}\text { South-eastern boundary } \\
\text { of UNISEL }\end{array}$ & $\begin{array}{l}3^{0} 24^{\prime} 54.73 " \mathrm{~N} \\
101^{0} 26^{\prime} 0.48^{\prime \prime} \mathrm{E}\end{array}$ & 6.430 & 22.19 & 5.30 & 12.90 \\
\hline SS5 & $\begin{array}{l}\text { Wetlands developed by } \\
\text { overflow of Pond } 1\end{array}$ & $\begin{array}{l}3^{0} 25^{\prime} 09.78^{\prime \prime} \mathrm{N} \\
101^{0} 25^{\prime} 59.41^{\prime \prime} \mathrm{E}\end{array}$ & 6.520 & 22.92 & 5.40 & 13.80 \\
\hline SS6 & Bank of Pond 1 & $\begin{array}{l}3^{0} 25^{\prime} 11.54^{\prime \prime} \mathrm{N} \\
101^{0} 26^{\prime} 07.44^{\prime \prime} \mathrm{E}\end{array}$ & 6.180 & 21.14 & 5.80 & 12.50 \\
\hline SS7 & $\begin{array}{l}\text { North-eastern } \\
\text { boundary of UNISEL }\end{array}$ & $\begin{array}{l}3^{0} 25^{\prime} 13.40^{\prime \prime} \mathrm{N} \\
101^{0} 26,11.64 " \mathrm{E}\end{array}$ & 6.340 & 22.81 & 5.70 & 13.60 \\
\hline SS8 & $\begin{array}{l}\text { Wetlands developed by } \\
\text { overflow of Pond } 1\end{array}$ & $\begin{array}{l}3^{0} 25^{\prime} 59.18^{\prime \prime} \mathrm{N} \\
101^{0} 25,56.90^{\prime \prime} \mathrm{E}\end{array}$ & 6.480 & 22.45 & 5.80 & 13.70 \\
\hline SS9 & $\begin{array}{l}\text { Junction of Sungai Ayer Hitam with } \\
\text { pond } 1 \text { on north-western side }\end{array}$ & $\begin{array}{l}3^{0} 25^{\prime} 19.80^{\prime \prime} \mathrm{N} \\
101^{0} 26^{\prime} 13.07 ” \mathrm{E}\end{array}$ & 6.440 & 21.91 & 5.80 & 14.10 \\
\hline SS10 & $\begin{array}{l}\text { Junction of Sungai Ayer Hitam } \\
\text { with pond } 1 \text { on south-western side }\end{array}$ & $\begin{array}{l}3^{0} 25^{\prime} 22.79^{\prime \prime} \mathrm{N} \\
101^{0} 26^{\prime} 11.06^{\prime \prime} \mathrm{E}\end{array}$ & 6.300 & 21.65 & 5.80 & 14.50 \\
\hline SS11 & $\begin{array}{l}\text { Wetland between } \\
\text { Pond } 1 \text { and Pond } 2\end{array}$ & $\begin{array}{l}3^{0} 25^{\prime} 20.64 " \mathrm{~N} \\
101^{0} 25,54.37^{\prime \prime} \mathrm{E}\end{array}$ & 6.380 & 22.24 & 5.40 & 14.80 \\
\hline SS12 & $\begin{array}{l}\text { Embankment } \\
\text { of Pond } 2\end{array}$ & $\begin{array}{l}3^{0} 25^{\prime} 27.52 ” \mathrm{~N} \\
101^{0} 25,53.89 ” \mathrm{E}\end{array}$ & 6.410 & 21.14 & 5.80 & 13.80 \\
\hline SS13 & $\begin{array}{l}\text { Embankment } \\
\text { of Pond } 2\end{array}$ & $\begin{array}{l}3^{0} 25^{\prime} 22.86 " \mathrm{~N} \\
101^{0} 25,51.67^{\prime \prime} \mathrm{E}\end{array}$ & 6.280 & 22.34 & 5.10 & 12.90 \\
\hline SS14 & $\begin{array}{l}\text { Embankment } \\
\text { of Pond } 2\end{array}$ & $\begin{array}{l}3^{0} 25^{\prime} 34.95^{\prime \prime} \mathrm{N} \\
101^{0} 25^{\prime} 49.93^{\prime \prime} \mathrm{E}\end{array}$ & 6.390 & 21.87 & 5.80 & 14.60 \\
\hline SS15 & $\begin{array}{l}\text { Embankment of } \\
\text { Pond } 2\end{array}$ & $\begin{array}{l}3^{0} 25^{\prime} 36.24 " \mathrm{~N} \\
101^{0} 25,52.14 " \mathrm{E}\end{array}$ & 6.430 & 22.39 & 5.70 & 13.30 \\
\hline Mean & $\mathrm{X}$ & 6.36 & 22.000 & 5.60 & 13.70 & \\
\hline $\begin{array}{l}\text { Standare } \\
\text { Variance }\end{array}$ & iation & Ơ & 0.090 & 0.61 & 0.23 & 0.84 \\
\hline Variance & ndard Deviation) & $\mathrm{O}^{2}$ & 0.009 & 0.37 & 0.05 & 0.71 \\
\hline
\end{tabular}

Table 5: Heavy metals concentration in the surface water of the study area

\begin{tabular}{|c|c|c|c|c|c|c|c|c|c|c|c|}
\hline \multirow{2}{*}{$\begin{array}{l}\text { Sample } \\
\text { No. }\end{array}$} & \multirow[b]{2}{*}{ Location } & \multirow[b]{2}{*}{ Coordinates } & \multicolumn{3}{|c|}{ Element Concentration mg/l (ppm) } & \multirow[b]{2}{*}{$\mathrm{Co}^{2+}$} & \multirow[b]{2}{*}{$\mathrm{As}^{3+}$} & \multirow[b]{2}{*}{$\mathrm{Cu}^{2+}$} & \multirow[b]{2}{*}{$\mathrm{Fe}^{2-}$} & \multirow[b]{2}{*}{$\mathrm{Mn}^{2+}$} & \multirow[b]{2}{*}{$\mathrm{Sn}^{2+}$} \\
\hline & & & $\mathrm{Pb}^{2+}$ & $\mathrm{Zn}^{2+}$ & $\mathrm{Ni}^{2+}$ & & & & & & \\
\hline WS1 & $\begin{array}{l}\text { Junction of Sungai Selangor } \\
+ \text { Sungai Ayer Hitam }\end{array}$ & $\begin{array}{l}3^{0} 24^{\prime} 28.04 " \mathrm{~N} \\
101^{0} 255^{\prime} 54.89 " \mathrm{E}\end{array}$ & 38.00 & 88.00 & 2.50 & 1.00 & 30.00 & 59.00 & 6.0 & 44.00 & 85.00 \\
\hline WS2 & $\begin{array}{l}\text { Junction of Sungai Ayer } \\
\text { Hitam + Sungai Udang }\end{array}$ & $\begin{array}{l}3^{0} 24^{\prime} 30.96^{\prime \prime} \mathrm{N} \\
101^{0} 25^{\prime} 54.08^{\prime \prime} \mathrm{E}\end{array}$ & 46.00 & 86.00 & 2.50 & 2.10 & 35.00 & 78.00 & 10.0 & 46.00 & 100.00 \\
\hline WS3 & $\begin{array}{l}\text { Sungai Ayer Hitam } \\
\text { water flow }\end{array}$ & $\begin{array}{l}3^{0} 24^{\prime} 36.54 " \mathrm{~N} \\
101^{0} 25,59.96 " \mathrm{E}\end{array}$ & 45.00 & 86.00 & 3.10 & 2.00 & 32.00 & 68.00 & 12.0 & 46.00 & 150.00 \\
\hline WS4 & $\begin{array}{l}\text { Sungai Ayer Hitam } \\
\text { at bank of UNISEL }\end{array}$ & $\begin{array}{l}3^{0} 24^{\prime} 56.68^{\prime \prime} \mathrm{N} \\
101^{0} 25^{\prime} 59.18^{\prime \prime} \mathrm{E}\end{array}$ & 51.00 & 87.00 & 3.60 & 1.90 & 36.00 & 76.00 & 15.0 & 47.00 & 150.00 \\
\hline WS5 & $\begin{array}{l}\text { Sungai Ayer Hitam } \\
\text { at bank of UNISEL }\end{array}$ & $\begin{array}{l}3^{0} 25^{\prime} 06.69^{\prime \prime} \mathrm{N} \\
101^{0} 26^{\prime} 08.14^{\prime \prime} \mathrm{E}\end{array}$ & 51.00 & 86.00 & 2.90 & 2.00 & 52.00 & 69.00 & 13.0 & 49.00 & 155.00 \\
\hline WS6 & $\begin{array}{l}\text { Junction of Pond } 1 \\
\text { to Sungai Ayer Hitam }\end{array}$ & $\begin{array}{l}3^{0} 25^{\prime} 16.57^{\prime \prime} \mathrm{N} \\
101^{0} 26^{\prime} 09.24^{\prime \prime} \mathrm{E}\end{array}$ & 60.00 & 88.00 & 7.50 & 2.90 & 78.00 & 71.00 & 10.0 & 49.00 & 200.00 \\
\hline WS7 & Pond 1 & $\begin{array}{l}3^{0} 25^{\prime} 13.37^{\prime \prime} \mathrm{N} \\
101^{0} 26^{\prime} 04.66^{\prime \prime} \mathrm{E}\end{array}$ & 58.00 & 88.00 & 8.10 & 2.50 & 91.00 & 60.00 & 12.0 & 48.00 & 225.00 \\
\hline WS8 & Pond 1 & $\begin{array}{l}3^{0} 25^{\prime} 15.56^{\prime \prime} \mathrm{N} \\
101^{0} 26^{\prime} 0.79 \text { " E }\end{array}$ & 89.00 & 90.00 & 6.20 & 2.80 & 88.00 & 80.00 & 15.0 & 49.00 & 268.00 \\
\hline WS9 & $\begin{array}{l}\text { Junction of Pond } 1 \text { to another Pond } \\
\text { Pond } 2 \text { at north-eastern boundary UNISEL }\end{array}$ & $\begin{array}{l}3^{0} 25^{\prime} 13.87^{\prime \prime} \mathrm{N} \\
101^{0} 25,55.35^{\prime \prime} \mathrm{E}\end{array}$ & 67.00 & 90.00 & 4.30 & 3.00 & 67.00 & 75.00 & 20.0 & 48.00 & 227.00 \\
\hline WS10 & $\begin{array}{l}\text { Junction of Sungai } \\
\text { Ayer Hitam to }\end{array}$ & $\begin{array}{l}3^{0} 25^{\prime} 20.92^{\prime \prime} \mathrm{N} \\
101^{0} 26^{\prime} 12.06^{\prime \prime} \mathrm{E}\end{array}$ & 80.00 & 92.00 & 3.40 & 2.70 & 91.00 & 70.00 & 14.0 & 48.00 & 199.00 \\
\hline WS11 & $\begin{array}{l}\text { Junction of Sungai } \\
\text { Ayer Hitam to Pond } 2\end{array}$ & $\begin{array}{l}3^{0} 25^{\prime} 22.11^{\prime \prime} \mathrm{N} \\
101^{0} 26^{\prime} 6.66^{\prime \prime} \mathrm{E}\end{array}$ & 89.00 & 94.00 & 5.90 & 2.90 & 69.00 & 78.00 & 18.0 & 49.00 & 134.00 \\
\hline WS12 & Pond 2 & $\begin{array}{l}3^{0} 25^{\prime} 22.54^{\prime \prime} \mathrm{N} \\
101^{0} 26^{\prime} 0.94^{\prime \prime} \mathrm{E}\end{array}$ & 91.00 & 132.00 & 8.10 & 1.80 & 90.00 & 95.00 & 19.0 & 51.00 & 155.00 \\
\hline WS13 & Pond 2 & $\begin{array}{l}3^{0} 25^{\prime} 22.05^{\prime \prime} \mathrm{N} \\
101^{0} 25,58.38^{\prime \prime} \mathrm{E}\end{array}$ & 87.00 & 110.00 & 6.20 & 2.10 & 89.00 & 81.00 & 20.0 & 50.00 & 190.00 \\
\hline WS14 & Pond2 & $\begin{array}{l}3^{0} 25^{\prime} 23.71^{\prime \prime} \mathrm{N} \\
101^{0} 25^{\prime} 52.42^{\prime \prime} \mathrm{E}\end{array}$ & 94.00 & 122.00 & 5.50 & 2.50 & 71.00 & 88.00 & 18.0 & 49.00 & 198.00 \\
\hline WS15 & $\begin{array}{l}\text { Junction of Pond } 2 \\
\text { to another Pond } \\
\text { Standard Deviation }\end{array}$ & $\begin{array}{l}3^{0} 25^{\prime} 33.21^{\prime \prime} \mathrm{N} \\
101^{0} 25^{\prime} 51.34^{\prime \prime} \mathrm{E} \\
-\end{array}$ & 96.00 & 121.00 & 2.80 & 1.80 & 77.00 & 80.00 & 16.0 & 48.00 & 250.00 \\
\hline Mean & Variance (Standard Deviation) & $\begin{array}{l}\mathrm{X} \\
\mathrm{O}^{\prime} \\
\mathrm{O}^{2}\end{array}$ & $\begin{array}{c}69.46 \\
20.7 \\
428.55\end{array}$ & $\begin{array}{r}87.8 \\
31.96 \\
1021.70\end{array}$ & $\begin{array}{l}4.80 \\
2.06 \\
4.24\end{array}$ & $\begin{array}{l}2.20 \\
0.55 \\
0.30\end{array}$ & $\begin{array}{r}66.00 \\
23.36 \\
546.11\end{array}$ & $\begin{array}{r}75.00 \\
9.56 \\
91.45\end{array}$ & $\begin{array}{r}14.00 \\
4.10 \\
16.83\end{array}$ & $\begin{array}{r}48.00 \\
1.75 \\
3.06\end{array}$ & $\begin{array}{r}179.00 \\
52.53 \\
2760.00\end{array}$ \\
\hline
\end{tabular}


Am. J. Environ. Sci., 7 (4): 383-396, 2011

Table 6: Heavy metals concentration in the soil of the study area

\begin{tabular}{|c|c|c|c|c|c|c|c|c|c|c|c|}
\hline \multirow{2}{*}{$\begin{array}{l}\text { Sample } \\
\text { No. }\end{array}$} & \multirow{2}{*}{ Location } & \multirow[b]{2}{*}{ Coordinates } & \multicolumn{3}{|c|}{ Element Concentration $\mathrm{mg} / \mathrm{kg}$} & \multirow[b]{2}{*}{$\mathrm{Co}^{2+}$} & \multirow[b]{2}{*}{$\mathrm{As}^{3+}$} & \multirow[b]{2}{*}{$\mathrm{Cu}^{2+}$} & \multirow[b]{2}{*}{$\mathrm{Fe}^{2-}$} & \multirow[b]{2}{*}{$\mathrm{Mn}^{2+}$} & \multirow[b]{2}{*}{$\mathrm{Sn}^{2+}$} \\
\hline & & & $\mathrm{Pb}^{2+}$ & $\mathrm{Zn}^{2+}$ & $\mathrm{Ni}^{2+}$ & & & & & & \\
\hline $\begin{array}{l}\text { SS1 } \\
\end{array}$ & $\begin{array}{l}\text { Junction of Jalan Timur } \\
\text { Tambahan + Sungai Selangor }\end{array}$ & $\begin{array}{l}3^{0} 24^{\prime} 29.80^{\prime \prime} \mathrm{N} \\
101^{0} 25^{\prime} 55.08^{\prime \prime} \mathrm{E}\end{array}$ & 110.00 & 120.00 & 8.50 & 3.00 & 70.0 & 120.00 & 22.0 & 84.00 & 425.00 \\
\hline SS2 & $\begin{array}{l}\text { Bank of Sungai Ayer } \\
\text { Hitam + Sungai Udang }\end{array}$ & $\begin{array}{l}3^{0} 24^{\prime} 32.03 \text { " N } \\
101^{\circ} 25^{\prime} 54.75^{\prime \prime} \mathrm{E}\end{array}$ & 96.00 & 113.00 & 5.50 & 2.80 & 75.0 & 112.00 & 24.0 & 91.00 & 400.00 \\
\hline SS3 & Bank of Sungai Ayer Hitam & $\begin{array}{l}3^{0} 24^{\prime} 36.29^{\prime \prime} \mathrm{N} \\
101^{\circ} 25^{\prime} 57.34^{\prime \prime} \mathrm{E}\end{array}$ & 110.00 & 132.00 & 6.10 & 2.00 & 82.0 & 128.00 & 25.0 & 72.00 & 390.00 \\
\hline SS4 & $\begin{array}{l}\text { South-eastern } \\
\text { boundary of UNISEL }\end{array}$ & $\begin{array}{l}3^{0} 24^{\prime} 54.73^{\prime \prime} \mathrm{N} \\
101^{\circ} 26^{\prime} 0.48^{\prime \prime} \mathrm{E}\end{array}$ & 115.00 & 110.00 & 6.60 & 2.90 & 86.0 & 135.00 & 25.0 & 84.00 & 350.00 \\
\hline SS5 & $\begin{array}{l}\text { Wetlands developed } \\
\text { by overflow of Pond } 1\end{array}$ & $\begin{array}{l}3^{0} 25^{\prime} 09.78^{\prime \prime} \mathrm{N} \\
101^{\circ} 25^{\prime} 59.41^{\prime \prime} \mathrm{E}\end{array}$ & 120.00 & 122.00 & 7.90 & 2.00 & 62.0 & 140.00 & 25.0 & 89.00 & 355.00 \\
\hline SS6 & Bank of Pond 1 & $\begin{array}{l}3^{0} 25^{\prime} 11.54^{\prime \prime} \mathrm{N} \\
101^{0} 26^{\prime} 07.44^{\prime \prime} \mathrm{E}\end{array}$ & 102.00 & 121.00 & 7.50 & 2.90 & 78.0 & 137.00 & 25.0 & 81.00 & 338.00 \\
\hline SS7 & $\begin{array}{l}\text { North-eastern } \\
\text { boundary of UNISEL }\end{array}$ & $\begin{array}{l}3^{0} 25^{\prime} 13.40^{\prime \prime} \mathrm{N} \\
101^{0} 26^{\prime} 11.64^{\prime \prime} \mathrm{E}\end{array}$ & 108.00 & 100.00 & 8.10 & 2.50 & 91.0 & 125.00 & 26.0 & 79.00 & 325.00 \\
\hline SS8 & $\begin{array}{l}\text { Wetlands developed } \\
\text { by overflow of Pond } 1\end{array}$ & $\begin{array}{l}3^{0} 25^{\prime} 59.18^{\prime \prime} \mathrm{N} \\
101^{0} 25^{\prime} 56.90^{\prime \prime} \mathrm{E}\end{array}$ & 99.00 & 120.00 & 6.20 & 2.80 & 88.0 & 100.00 & 26.0 & 86.00 & 368.00 \\
\hline SS9 & $\begin{array}{l}\text { Junction of Sungai Ayer Hitam } \\
\text { with pond } 1 \text { on north-western side }\end{array}$ & $\begin{array}{l}3^{0} 25^{\prime} 19.80^{\prime \prime} \mathrm{N} \\
101^{0} 26^{\prime} 13.07 " \mathrm{E}\end{array}$ & 97.00 & 102.00 & 7.30 & 3.00 & 67.0 & 125.00 & 28.0 & 98.00 & 387.00 \\
\hline SS10 & $\begin{array}{l}\text { Junction of Sungai Ayer Hitam } \\
\text { with pond } 1 \text { on south-western side }\end{array}$ & $\begin{array}{l}3^{0} 25^{\prime} 22.79^{\prime \prime} \mathrm{N} \\
101^{0} 26^{\prime} 11.06^{\prime \prime} \mathrm{E}\end{array}$ & 120.00 & 112.00 & 6.40 & 2.70 & 91.0 & 120.00 & 25.0 & 98.00 & 399.00 \\
\hline SS11 & $\begin{array}{l}\text { Wetland between Pond } 1 \text { and } \\
\text { Pond } 2\end{array}$ & $\begin{array}{l}3^{0} 25^{\prime} 20.64 ” \mathrm{~N} \\
101^{0} 25^{\prime} 54.37^{\prime \prime} \mathrm{E}\end{array}$ & 85.00 & 100.00 & 5.90 & 2.90 & 69.0 & 128.00 & 26.0 & 81.00 & 434.00 \\
\hline SS12 & Embankment of Pond 2 & $\begin{array}{l}3^{0} 25^{\prime} 27.52^{\prime \prime} \mathrm{N} \\
101^{0} 25^{\prime} 53.89^{\prime \prime} \mathrm{E}\end{array}$ & 99.00 & 132.00 & 8.10 & 2.80 & 90.0 & 125.00 & 25.0 & 83.00 & 455.00 \\
\hline SS13 & Embankment of Pond 2 & $\begin{array}{l}3^{0} 25^{\prime} 22.86 " \mathrm{~N} \\
101^{0} 25^{\prime} 51.67^{\prime \prime} \mathrm{E}\end{array}$ & 97.00 & 110.00 & 6.20 & 2.10 & 89.0 & 130.00 & 28.0 & 90.00 & 490.00 \\
\hline SS14 & Embankment of Pond 2 & $\begin{array}{l}3^{0} 25^{\prime} 34.95^{\prime \prime} \mathrm{N} \\
101^{0} 25^{\prime} 49.93^{\prime \prime} \mathrm{E}\end{array}$ & 110.00 & 122.00 & 5.50 & 3.50 & 71.0 & 128.00 & 24.0 & 81.00 & 498.00 \\
\hline SS15 & $\begin{array}{l}\text { Embankment of Pond } 2 \\
\text { Standard Deviation }\end{array}$ & $\begin{array}{l}3^{0} 25^{\prime} 36.24 ” \mathrm{~N} \\
101^{0} 25^{\prime} 52.14^{\prime \prime} \mathrm{E}\end{array}$ & 110.00 & 121.00 & 5.80 & 2.80 & 77.0 & 130.00 & 29.0 & 86.00 & 450.00 \\
\hline Mean & Variance (Standard Deviation) & $\begin{array}{l}\mathrm{X} \\
\mathrm{O}^{\prime} \\
\mathrm{O}^{2}\end{array}$ & $\begin{array}{r}105.00 \\
9.81 \\
96.31\end{array}$ & $\begin{array}{r}115.00 \\
10.24 \\
105.00\end{array}$ & $\begin{array}{l}6.70 \\
1.02 \\
1.05\end{array}$ & $\begin{array}{l}2.70 \\
0.41 \\
0.14\end{array}$ & $\begin{array}{r}79.0 \\
9.8 \\
96.2\end{array}$ & $\begin{array}{r}125.00 \\
9.94 \\
98.98\end{array}$ & $\begin{array}{r}25.50 \\
1.76 \\
3.12\end{array}$ & $\begin{array}{r}85.00 \\
6.92 \\
47.98\end{array}$ & $\begin{array}{r}404.00 \\
53.46 \\
2858.00\end{array}$ \\
\hline
\end{tabular}

Contribution of heavy metals in soil

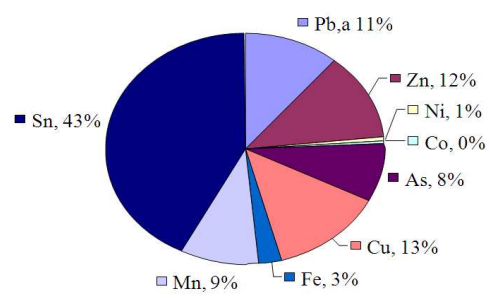

$\square \mathrm{Pb} \square \mathrm{Zn} \square \mathrm{Ni} \square \mathrm{Co} \square \mathrm{As} \square \mathrm{Cu} \square \mathrm{Fe} \square \mathrm{Mn} \square \mathrm{Sn}$

Fig. 5: Contribution of heavy metals in soil

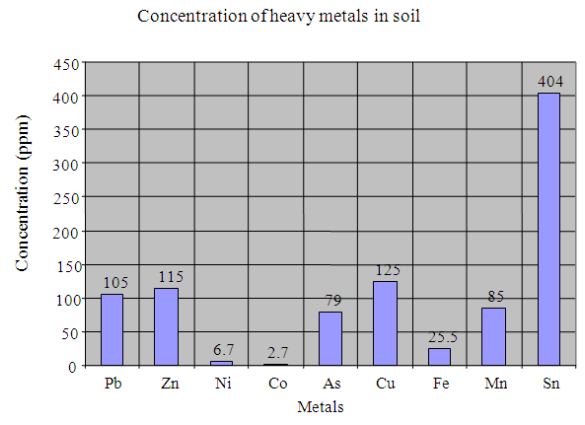

Fig. 6: Concentration of heavy metals in soil

\section{DISCUSSION}

According to results, there is variation in water quality at all sampling stations. The variation trends at all sampling stations are from upstream to downstream. Possible factors involved in this variation may include formation of wetlands, palm oil plantation and the dilution factor of water. Acidic $\mathrm{pH}$ and low $\mathrm{DO}$ is the characteristic of peat swamp water (flowing into the catchment) and also by metal and sand mining activity. The high conductivity values represent high concentration of total dissolved solids. The main source of high TDS value is the recent sand mining activity going on in the study area. This study shows that the water quality is degraded in the area.

According to values presented in Table 3, shows that the contents of the soil represent medium textured sandy soil. Sandy soils have low clay and organic matter contents and aggregation is very weak to nonexistent. The structure is called single grained. Such kind of soil cannot retain so much water and can drain quickly. Single drained soils required frequent irrigation and fertilization for plants roots to penetrate. Table 4 represents the physio-chemical characteristics of soil which indicates that soil temperature and hydraulic conductivity is feasible for plant growth but 
low $\mathrm{pH}$ due to high cations in soil and moisture content due to sandy structure depress plant growth. Similarly metal concentration of water and soil shows variation trends of decrease in metal concentration from upstream to downstreaam.

Wetland development conceptual model: (Table 1) shows the required construction materials quantities and estimated costs for the development of Bestari Jaya wetland. The estimated costs is RM57000, which is very physible and economic for this kind of system. In designing a treatment wetland for heavy metals removal, processes within three compartments must be considered: (1) water, (2) media and (3) biota (Sonntag and Cole, 2008; Hoehn et al., 2003). As outlined in (Fig. 7), water is the most essential compartment; all the processes of heavy metals removal within the other compartments are encompassed within the water compartment. Metal transport is dependent upon water movement which transports the heavy metals throughout the system.

The water causes the saturation of the media, allowing reduction of the redox value in order for treatment processes to occur within the media and related biota.

Each is dependent upon the other, with water as the most important compartment. The media compartment consists of any substrate used to contain both the water and the biota. For metals removal, the media used must act as an adsorbent for metal species.

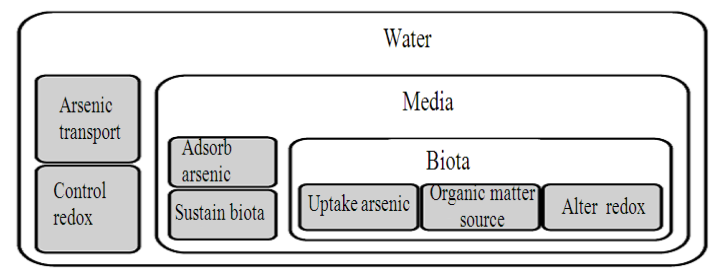

Fig. 7: Diagram noting the three compartments of a treatment wetland: water, soil and biota

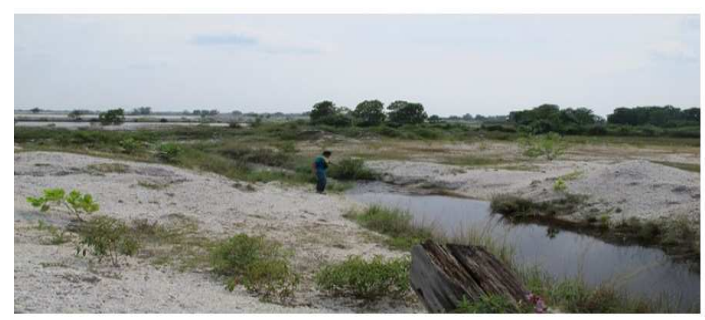

Fig. 8: View of reference Pond1. The end of inlet swale is visible in the foreground; the outlet is located on the opposite bank
The ability of the media to adsorb heavy metals is dependent upon its chemical make-up, and the species of arsenic present. Media within a treatment wetland can be selected to treat different concentrations or volumes of heavy metals. The media also sustains the biota, providing water and nutrients to both plants and microorganisms. The biota compartment consists of both plants and microorganisms. Biota can uptake heavy metals and alters the redox of the environment by several means. The biota is alsoa source of organic matter for the media used to better adsorb arsenic (Mitsch et al., 2008).

Biota used within a treatment wetland can be altered to best suit the conditions present.

Proposed design for Bestari Jaya wetland: Wetland system was proposed on the grounds of the Bestari Jaya mine tailings.

The system comprises of three compartments in series-an 'inflow' pond receiving untreated tailings water overflowing into a wetland compartment, which in turn overflows into an 'outflow' pond receiving the now treated water (Fig. 8). Waterproof baffles in each wetland compartment serve to increase the flow path of the water, thereby increasing the potential for sulphate retention. On site a computer (ACS Pentium PC) connected to the pumps regulates the flow of tailings water through the systems. Also connected to the computer are four permanent industrial-grade electrodes (Rosemount Solu Cube ${ }^{\circledR}$ Analyser Model 2700), one situated in each of the four ponds. These facilitate continuous and simultaneous monitoring of conductivity and temperature. Data are logged into a database, every half-hour for the initial two months, thereafter every three hours, $24 \mathrm{~h}$ a day, and can be accessed remotely via a portable modem. This makes it possible to monitor the performance of the systems from our laboratory at the University of Malaya using pc ANYWHERE 32 software.

Reference pond 1: Wetland is developed, by following the treatment volume approach and comprises five ponds (referred to as P1, P2, P3, P4 and P5) lined by compacted clay and separated by shallow vegetated areas submerged in wet conditions. Reference Pond is located at $3^{\circ} 26^{\prime} 11.10 \mathrm{~N}, 101^{\circ} 26^{\prime} 20.32 \mathrm{E}$, Elevation 7 $\mathrm{m}$. This pond is $0.8 \mathrm{~km}$ from the UNISEL and Bestari Jaya Town and was selected according to its position with reference to Selangor River and water flow from the catchment. The pond has an area of $2200 \mathrm{~m}^{2}$, a maximum depth of $8.5 \mathrm{~m}$ in the centre and volume of $1500 \mathrm{~m}^{3}$ (Fig. 9). Pond is estimated to receive $105 \mathrm{~m}^{3}$ $\mathrm{h}^{-1}$ (i.e., a Vt of $1840 \mathrm{~m}^{3}$ ) runoff water from mining 
area including tin tailing and sand mining water with high TDS and TSS. Wastewater from the mining ponds and runoff from tin tailings will discharge into a swale (45 $\mathrm{m}$ long) from two pipes (50 and $80 \mathrm{~cm}$ in diameter) over paving slabs to minimise erosion. Water leaves reference pond $\mathrm{P} 1$ runs through a long shallow vegetated area (c. $40 \mathrm{~m}$ long, $15 \mathrm{~m}$ wide) and through a series of three ponds (P2: $115 \mathrm{~m}^{2}, \mathrm{P} 3: 105 \mathrm{~m}^{2}, \mathrm{P} 4: 190$ $\mathrm{m}^{2}$, up to $1 \mathrm{~m}$ deep) separated by short (c. $20 \mathrm{~m}$ ) shallow vegetated (grass or watercress) areas.

Flow then enters a large and deep pond (P5, c. $2500 \mathrm{~m}^{2}$, up to $1.5 \mathrm{~m}$ deep, vegetated) (Fig. 10 and 11). Finally, under normal conditions, water will leave pond P5 through an inlet located on the south-east corner of the pond and flows into a ditch transferring treated water to River Ayer Hitam that ultimately fed up into river Selangor. The wetland compartments will be planted with Typha latifolia (four plants per $\mathrm{m}^{2}$ ) and Phragmites australis (nine plants per $\mathrm{m}^{2}$ ).

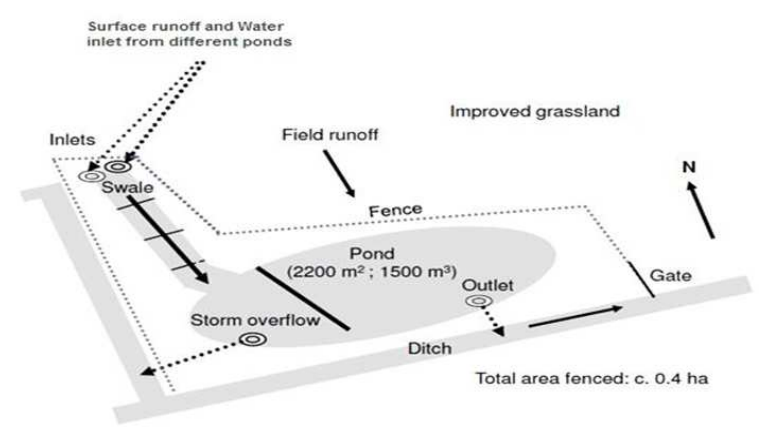

Fig. 9: Proposed Sketch of Reference Pond 1 (not to scale) dashed arrows represent flow in subsurface pipes and full arrows represent surface flow

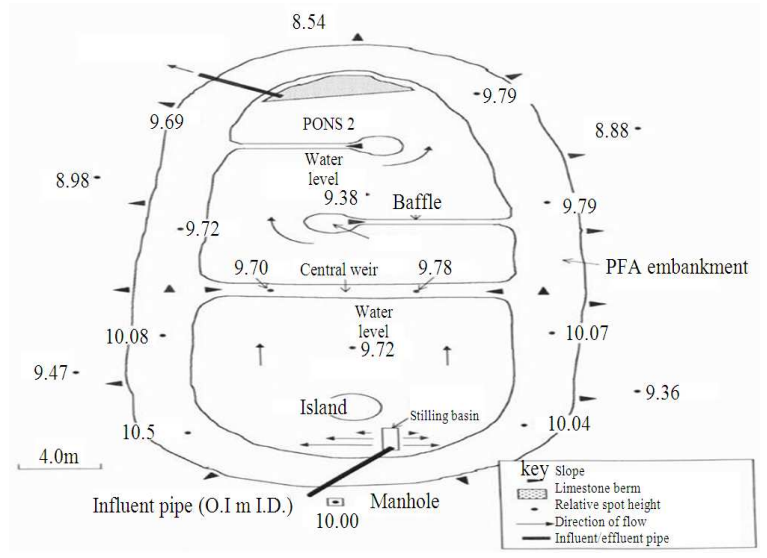

Fig. 10: Proposed Structure with construction materials for Pond 1 and Pond 2
Glyceria fluitans (seven plants per $\mathrm{m} 2$ ), bulbs of Iris pseudacorus (five plants per $\mathrm{m}^{2}$, rhizomes of Typha latifolia (six plants per $\mathrm{m}^{2}$ ) Juncus effusus (seven plants per $\mathrm{m}^{2}$ ), Phalaris arundinacea (nine plants per $\mathrm{m}^{2}$ ) and Cyperus rotuduss L. (nine plants per $\mathrm{m}^{2}$ ). Flow rates were set at $300-500 \mathrm{~mL} \min ^{-1}$. These rates were adapted to fit the size of the systems based on the values given for other operational systems as described by Crites (1994). The theoretical residence time for the systems is 52 days, but the applicable value has yet to be confirmed. Here we report a select number of parameters measured, namely, volunteer species (invaders), $\mathrm{pH}$, redox potential, conductivity and sulphate concentrations in water.

The site was visited on a monthly or bi-weekly basis and some parameters were monitored continuously. The $\mathrm{pH}$ was measured using a glass combination electrode connected to a pH meter (WTW pH90). Redox potential was measured using a platinum electrode connected to a $\mathrm{mV}$ meter (WTW pH90). Conductivity was measured using the industrial elecelectrodes mentioned above. For the analysis of sulphate a Dionex ion chromatogram was used. Invading flora will be identified using the standard key of the Malaysian Botanical Society.

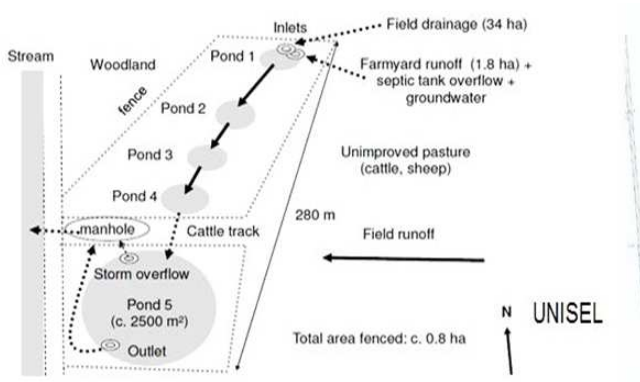

(a)

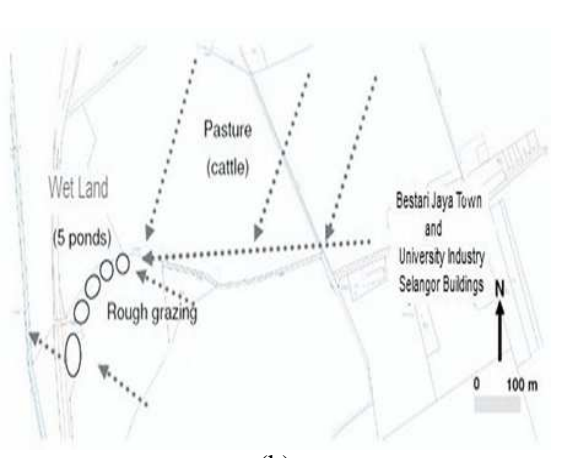

(b)

Fig. 11: Proposed sketch of wetland ponds (not to scale) at two different angles. Dashed arrows represent $\mathrm{t}$ underground piped flow and full arrows represent surface flow 
The wetland compartment of each system is filled with approximately $50 \mathrm{~cm}$ depth of a mixture of cattle manure $(25 \%)$ and municipal waste compost $(75 \%)$. This mixture was chosen because literature shows that it combined good permeability with optimal growth of plants. At the bottom of the inflow and outflow ponds in each system, a layer of about $25 \mathrm{~cm}$ of a 1:6 mixture of cattle manure and municipal waste compost was deposited to provide a substrate for the invertebrate species that spontaneously inhabit the systems. The planting density chosen was based on similar research on constructed wetlands (Yang et al., 2006). Additionally 30 tonnes of limestone are deposited at the far end of the wetland, to facilitate final $\mathrm{pH}$ adjustment if it should be required. (Fig. 10) shows placement of limestone at the far end of the treatment system. The system was designed such that the compost depth in the wetland would be $0.30-0.50 \mathrm{~m}$. An additional $0.30 \mathrm{~m}$ of freeboard is allowed for accumulation of material on the substrate surface. The total area of substrate surface is $440 \mathrm{~m}^{2}$. To generate additional hydraulic head a concrete wall will construct across the culvert from which the discharge emanates. Two sections of $100 \mathrm{~mm}$ diameter pipe were built into this wall. The first carries water underground to the influent point of the wetland, discharging into a basin from where the water is distributed across the wetland. The second section of pipe allows overflow back into the original watercourse when flow rates exceed approximately 400 litres $\mathrm{min}^{-1}$. Because pollutant concentrations are lower at higher flow-rates due to dilution, and because of further dilution of the overflow water by the effluent from the wetland, the impact of this water on the receiving watercourse is minimal. The water outlet structure was originally a section of $150 \mathrm{~mm}$ diameter plastic pipe buried into the retaining embankment. A movable $90^{\circ}$ bend on the wetland-side of this pipe allowed the water level in the wetland to be adjusted (although typically the water level has been maintained approximately 50$100 \mathrm{~mm}$ above the surface of the substrate (Fig. 10 and 11).

Because the site slopes downwards slightly (away from the proposed influent point to the wetland), a central weir was incorporated in the design in order that the wetland could be constructed on two levels, the second cell being $0.4 \mathrm{~m}$ lower than the first cell. In this way savings were made in terms of both materials costs and land area used for the embankment.

The quantities of materials used, and the overall estimated cost for the development of wetland at Bestari Jaya is given below in the Table.

\section{CONCLUSION}

The preliminary result obtained from this study is alarming. The results of water quality trends clearly show that majority of water quality parameters are quite high and fall in Class III in terms of Malaysian Interim Water Quality Standards. The picture is more severe if we talk in terms of heavy metals concentration in the area. It falls above level IV in INWQS. After comparison of different parts of study area it is concluded that Bestari Jaya catchment has high pollution risks on environment, Sungai Ayer Hitam recipient of catchment water is highly polluted river that ultimately ends into Sungai Selangor, is vulnerable and sensitive ecosystem especially to metal pollution. Therefore lot of research needs to be carried out to access the pollution impact of the area on the environment and for the rehabilitation and reclamation steps to be taken.Wetlands have a strong capacity for the retention of pollutants, including those originating from mining activities. The establishment of a wetland cover over tailings provides a promising alternative to the more traditional dry land option. Critics of both applications frequently doubt the longevity of these systems. Where the use of wetlands for treatment of polluted water is concerned the answer is simple-size matters. If a wetland is built sufficiently large to manage the input of pollutants, then it should be functional for many decades (Withey and Kooten, 2011).

Restriction in the use of wetlands for treatment of wastewater is therefore determined by the available space for construction of such a system. Revegetation of tailings with wetlands should be sustainable for indefinite periods of time. The vegetation component provides the source of organic matter needed to drive the chemical reduction of sulphides and the subsequent precipitation of metal sulphides (Ningthoujam et al., 2009). Through these processes the metals and sulphates are returned to the form they were derived from originally in the mining process, as many metal ores are sulphide in nature. Therefore, wetlands can be used to complete the recycling of mine wastes from sulphides back to sulphides:

- The proposed wetland at Bestari Jaya is comparatively small in engineering terms and is unique in the sense that the proposed wetland will play an important role in guiding the design of such systems for mining sites in Malaysia in future

- The wetland built is an anaerobic (compost) surface flow system. In engineering terms the decision to construct such a system was based on the limited hydraulic head available across the site 
Am. J. Environ. Sci., 7 (4): 383-396, 2011

- 640 tonnes of Pulverised Fuel Ash (PFA) were used for the construction of the retaining embankments, which are keyed into the in situ soil to a depth of approximately $0.2 \mathrm{~m}$. Extensive excavation of the in situ soil was not possible as it was found to be heavily contaminated with metals from former mining operations. The substrate of the wetland is a combination of horse manure, cow manure and municipal waste

- It is unclear whether temperature is the direct cause of changes in removal efficiency, or whether perhaps temperature changes influence microbial activity, which in turn affect metal removal efficiency. In either case this observation has some important implications. In particular, it suggests that wetland systems operating under cold climate conditions may be less effective, at least in terms of aluminium removal

- Three methods are currently in use for assessing constructed wetland performance

- Treatment efficiency (\%)

- Area-adjustedr emoval rates $(\mathrm{g} / \mathrm{m} 2 / \mathrm{d})$

- First-order removal constants $(\mathrm{m} / \mathrm{d})$

To make useful comparisons between constructed wetland systems a performance indicator must be independent of differences in influent pollutant concentration (Kadlec, 2006) A new method of wetland performance assessment proposed by (Kadlec, 2009) based on first-order removal of contaminants, appears to be a far better method of assessment.

Recommendations: Much can be drawn from the design of Bestari Jaya wetland and these lessons may be of considerable use for future constructed wetland projects:

A thorough characterisation of the quantity and quality of mine water to be treated proved essential in this project. There is no doubt that a similar familiarity should be encouraged for all such projects, since mine waters commonly exhibit fluctuations in both quantity and quality.

A key objective of the feasibility study was to design a treatment system that would be inexpensive in terms of both initial installation costs and long-term operating and maintenance costs. Investigation of the variety of construction materials available is therefore to be encouraged. Almost 50\% of the total expenditure of this project is on plant hire and operation. Typically costs are incurred even when machinery is not operating due to inclement weather conditions.
Establishing the exact removal mechanisms operational within the Bestari Jaya wetland will require detailed and long-term biogeochemical research, which was beyond the scope of this particular study. From the results of this work it would seem that particular emphasis needs to be placed on establishing the main mineral phases within the wetland substrate, and ascertaining the role of iron and sulphur cycling in the vicinity of the water-sediment interface.

If contaminant removal is rate dependent, as the weight of evidence suggests it is, then it is crucial to have accurate indications of residence times to properly understand the removal mechanisms operating within constructed wetlands. Tracer tests, using a conservative ion such as lithium, should be undertaken to achieve this. However, multiple tests would be required to establish residence times at different influent flowrates. The use of automatic sampling equipment would be of great use in this regard.

The first-order removal model of assessment proposed by (Kadlec, 2006) appears to be the most appropriate method for comparing wetland performance. This being the case, future constructed wetlands may be more effectively designed on the basis of the first-order removal model. However, as (Kadlec, 2009) point out, if this is to be possible future research must be undertaken to gather values for the first-order removal constant at constructed wetlands already operational.

The anaerobic wetland treatment appear to be a very promising new treatment technology, particularly for remediation of marginally polluted mine water discharges. Previously, no research has been undertaken to determine the mineral phases accreting to the media within the reactors. Such work would certainly assist in ascertaining the exact removal mechanisms operational in these treatment units. It appears that at full-scale a very efficient water distribution system would be required for the system to operate effectively.

Wetland/passive treatment of other waste streams may be feasible, and in some cases has been successfully undertaken. Elements of the research presented here may be applicable to other water pollution issues, and an investigation of such possibilities might prove fruitful. In particular the following types of wastes may be suitable for passive treatment of landfill drainage, airport/runway drainage and sewage effluent and railway runoff.

\section{ACKNOWLEDGEMENT}

The study reported in this study is a basic research work carried out at Analytical Laboratory, Department 
Am. J. Environ. Sci., 7 (4): 383-396, 2011

of Chemistry and partially at Department of Geology, University of Malaya. I take this opportunity to express my gratitude to Ministry of Higher Education, Malaysia to provide me scholarship to complete my higher studies in Malaysia. Thanks also go to Institut Pengurusan Dan Pemantauan Penyelidikan, (IPPP) University of Malaya to provide me enough funding (Project No. PS355/2009C) for the completion of this valuable research.

\section{REFERENCES}

Ahmadpour, P., A.M. Nawi, A. Abdu, H. AbdulHamid and D.K. Singh et al., 2010. Uptake of heavy metals by Jatropha curcas L. planted in soils containing sewage sludge. Am. J. Applied Sci., 7: 1291-1299. DOI: 10.3844/ajassp.2010.1291.1299

Al-Hamed, S.A., S.A. Al-Suhaibani, F.S. Mohammad and M.F.I. Wahby, 2010. Development of a comprehensive computer program for predicting farm energy. Am. J. Agric. Biol. Sci., 5: 89-101. DOI: 10.3844/ajabssp.2010.89.101

Amezaga, J.M., L. Santamaria and A.J. Green, 2002. Biotic wetland connectivity-supporting a new approach for wetland policy. Acta Oecol., 23: 213222. DOI: $10.1016 / \mathrm{S} 1146-609 \mathrm{X}(02) 01152-9$

Asadi, A., Y. Mohammadi and H.S. Fami, 2009. Investigation of the agricultural water management mechanisms in zarindasht county, fars province, Iran. Am. J. Agric. Biol. Sci., 4: 110-117. DOI: 10.3844/ajabssp.2009.110.117

Ashraf, M.A., M. J. Maah, I. Yusoff, A. Wajid and K. Mahmood, 2011b. Sand mining effects, causes and concerns: A case study from Bestari Jaya, Selangor, Peninsular Malaysia. Sci. Res. Essays, 6: 1216-1231.

Ashraf, M.A., M.J. Maah and I. Yusoff, 2011a. Heavy metals accumulation in plants growing on former tin mining catchment. International J. Environ. Sci. Technol., 8: 401-416.

Brooks, R.P., D.H. Wardrop, C.A. Cole, D.A. Campbell and D.A. Campbell, 2005. Are we purveyors of wetland homogeneity?: A model of degradation and restoration to improve wetland mitigation performance. Ecol. Eng., 24: 331-340. DOI: 10.1016/j.ecoleng.2004.07.009

Cvetkovic, M. and P. Chow-Fraser, 2011. Use of ecological indicators to assess the quality of Great Lakes coastal wetlands. Ecol. Indicators, 11: 16091622. DOI: 10.1016/j.ecolind.2011.04.005

Faulwetter, J.L., V. Gagnon, C. Sundberg, F. Chazarenc and M.D. Burr et al., 2008. Microbial processes influencing performance of treatment wetlands: A review. Ecol. Eng., 35: 987-1004. DOI: 10.1016/j.ecoleng.2008.12.030

Galbrand, C.C., A.M. Snow, A.E. Ghaly and R. Cote, 2008. Establishment and evaluation of the vegetative community in a surface flow constructed wetland treating industrial park contaminants. Am. J. Agric. Biol. Sci., 3: 417-432. DOI: $10.3844 /$ ajabssp.2008.417.432

Ghaly, A., B. Zhang and D. Dave, 2011. Biodegradation of phenolic compounds in creosote treated wood waste by a composting microbial culture augmented with the fungus Thermoascus aurantiacus. Am. J. Biochem. Biotechnol., 7: 90103. DOI: 10.3844/ajbbsp.2011.90.103

Heryati, Y., A. Abdu, M.N. Mahat, H. Abdul-Hamid and S. Jusop et al., 2011. Comparing the fertility of soils under khaya ivorensis plantation and regenerated degraded secondary forests. Am. J. Applied Sci., 8: 472-480. DOI: 10.3844/ajassp.2011.472.480

Hoehn, J.P., F. Lupi and M.D. Kaplowitz, 2003. Untying a Lancastrian bundle: Valuing ecosystems and ecosystem services for wetland mitigation. J. Environ. Manage., 68: 263-272. DOI: 10.1016/S0301-4797(03)00069-0

Jitrapinate, N., V. Sriboonlue, K. Srisuk and D. Hormdee, 2011. Simplified procedure for unsaturated flow parameters. Am. J. Applied Sci., 8: 635-643. DOI: 10.3844/ajassp.2011.635.643

Kadlec R.H., 2006. Free surface wetlands for phosphorus removal: The position of the everglades nutrient removal project. Ecol. Eng., 27: 361-379. DOI: 10.1016/j.ecoleng.2006.05.019

Kadlec, R.H., 2009. Comparison of free water and horizontal subsurface treatment wetlands. Ecol. Eng., 35: 159-174. DOI: 10.1016/j.ecoleng.2008.04.008

Kadlec, R.H., C. Cuvellier and T. Stober, 2010. Performance of the Columbia, Missouri, treatment wetland. Ecol. Eng., 36: 672-684. DOI: 10.1016/j.ecoleng.2009.12.009

Kangrang, A., and C. Chaleeraktrakoon, 2007. A fuzzygas model for determining varied irrigation efficiency. Am. J. Applied Sci., 4: 339-345. DOI: 10.3844/ajassp.2007.339.345

Keita, S., Z. Tang, S. Dong and Y. Jiang, 2009. Evaluation of some organic pollutants transport into the shallow groundwater and surface water of jiaxing landfill area. Am. J. Applied Sci., 6: 20102017. DOI: 10.3844/ajassp.2009.2010.2017

Knight, R.L., V.W.E. Payne, R.E. Borer, R.A. Clarke and J.H Pries, 2000. Constructed wetlands for livestock wastewater management. Ecol. Eng., 15: 41-55. DOI: 10.1016/S0925-8574(99)00034-8 
Knowles, P., G. Dotro, J. Nivala and J. Garcia, 2011. Clogging in subsurface-flow treatment wetlands: Occurrence and contributing factors. Ecol. Eng., 37: 99-112. DOI: 10.1016/j.ecoleng.2010.08.005

Lang, M.W., E.S. Kasischke, S.D. Prince and K.W. Pittman, 2008. Assessment of C-band synthetic aperture radar data for mapping and monitoring Coastal Plain forested wetlands in the Mid-Atlantic Region, U.S.A. Remote Sens. Environ., 112: 41204130. DOI: $10.1016 /$ j.rse.2007.08.026

Michailidis, A., 2006. Impact analysis of irrigation projects: An application of contingent valuation method. Am. J. Agric. Biol. Sci., 1: 17-21. DOI: 10.3844/ajabssp.2006.17.21

Mitsch, W.J., J. Tejada, A. Nahlik, B. Kohlmann and B. Bernal et al., 2008. Tropical wetlands for climate change research, water quality management and conservation education on a university campus in Costa Rica. Ecol. Eng., 34: 276-288. DOI: 10.1016/j.ecoleng.2008.07.012

Nasipuri, P., G.G. Pandit, A.R. Thakur and S.R. Chaudhuri, 2010. Microbial consortia from taptapani hot water springs for mining effluent treatment. Am. J. Microbiol., 1: 23-29. DOI: 10.3844/ajmsp.2010.23.29

Ningthoujam, D.S., S. Sanasam and S. Nimaichand, 2009. Screening of actinomycete isolates from niche habitats in Manipur for antibiotic activity. Am. J. Biochem. Biotechnol., 5: 221-225. DOI: 10.3844/ajbbsp.2009.221.225

Noble, B., M. Hill and J. Nielsen, 2011. Environmental assessment framework for identifying and mitigating the effects of linear development to wetlands. Landscape Urban Plann., 99: 133-140. DOI: 10.1016/j.landurbplan.2010.09.004

Reza, M.M., 2008. Assessment of suspended sediments concentration in surface waters, using modis images. Am. J. Applied Sci., 5: 798-804. DOI: 10.3844/ajassp.2008.798.804

Simonit, S., F. Cattaneo and C. Perrings, 2005. Modelling the hydrological externalities of agriculture in wetlands: The case of rice in Esteros del Iberà, Argentina. Ecol. Modell., 186: 123-141. DOI: 10.1016/j.ecolmodel.2005.01.053

Sonntag, D.H., and C.A. Cole, 2008. Determining the feasibility and cost of an ecologically-based design for a mitigation wetland in central Pennsylvania, USA. Landscape Urban Plann., 87: 10-21. DOI: 10.1016/j.landurbplan.2008.03.008
Spieles, D.J., and W.J. Mitsch, 1999. The effects of season and hydrologic and chemical loading on nitrate retention in constructed wetlands: a comparison of low- and high-nutrient riverine systems. Ecol. Eng., 14: 77-91. DOI: 10.1016/S0925-8574(99)00021-X

Steer, D., T. Aseltyne and L. Fraser, 2003. Life-cycle economic model of small treatment wetlands for domestic wastewater disposal. Ecol. Econ., 44: 359-369. DOI: 10.1016/S0921-8009(03)00003-X

Tilley, D.R. and M.T. Brown, 1998. Wetland networks for stormwater management in subtropical urban watersheds. Ecol. Eng., 10: 131-158. DOI: 10.1016/S0925-8574(98)00010-X

Turner, R.K., J.C.J.M. van den Bergh, T. Soderqvist, A. Barendregt and J.V.D. Straaten et al., 2000. Ecological-economic analysis of wetlands: Scientific integration for management and policy. Ecol. Econ., 35: 7-23. DOI: 10.1016/S09218009(00)00164-6

Vymazal, J., 2009. The use constructed wetlands with horizontal sub-surface flow for various types of wastewater. Ecol. Eng., 35: 1-17. DOI: 10.1016/j.ecoleng.2008.08.016

Whigham, D.F., 1999. Ecological issues related to wetland preservation, restoration, creation and assessment. Sci. Total Environ., 240: 31-40. DOI: 10.1016/S0048-9697(99)00321-6

Withey, P. and G.C.V. Kooten, 2011. The effect of climate change on optimal wetlands and waterfowl management in Western Canada. Ecol. Econ., 70: 798-805. DOI: 10.1016/j.ecolecon.2010.11.019

Yang, B., C.Y. Lan, C.S. Yang, W.B. Liao and H. Chang et al., 2006. Long-term efficiency and stability of wetlands for treating wastewater of a lead/zinc mine and the concurrent ecosystem development. Environ. Pollut., 143: 499-512. DOI: 10.1016/j.envpol.2005.11.045

Zhang, B., A. Shahbazi, L. Wang, O. Diallo and A. Whitmore, 2010. Alkali pretreatment and enzymatic hydrolysis of cattails from constructed wetlands. Am. J. Eng. Applied Sci., 3: 328-332. DOI: 10.3844 ajeassp.2010.328.332 